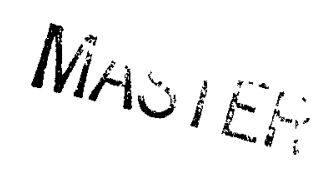

\title{
POWER BURSTS IN NUCLEAR REACTORS
}

H. C. CORBEN

Prepared under Contract AT(04-3)-165

With the United States Atomic Energy Commission

Electronic Research Laboratory THE RAMO-WOOLDRIDGE CORPORATION

Los Angeles, California

September 4, 1958 


\section{DISCLAIMER}

This report was prepared as an account of work sponsored by an agency of the United States Government. Neither the United States Government nor any agency Thereof, nor any of their employees, makes any warranty, express or implied, or assumes any legal liability or responsibility for the accuracy, completeness, or usefulness of any information, apparatus, product, or process disclosed, or represents that its use would not infringe privately owned rights. Reference herein to any specific commercial product, process, or service by trade name, trademark, manufacturer, or otherwise does not necessarily constitute or imply its endorsement, recommendation, or favoring by the United States Government or any agency thereof. The views and opinions of authors expressed herein do not necessarily state or reflect those of the United States Government or any agency thereof. 


\section{DISCLAIMER}

Portions of this document may be illegible in electronic image products. Images are produced from the best available original document. 
LEGAL NOTICE

This report was prepared as an account of Government sponsored work. Neither the United States, nor the Commission, nor any person acting on behalf of the Commission:

A. Makes any warranty or representation, express or implied, with respect to the accuracy, completeness, or usefulness of the information contained in this report, or that the use of any information, apparatus, method, or process disclosed in this report may not infringe privately owned rights; or

B. Assumes any liabilities with respect to the use of, or for damages resulting from the use of any information, apparatus, method, or process disclosed in this report.

As used in the above, "person acting on behalf of the Commission" includes any employee or contractor of the Commission to the extent that such employee or contractor prepares, handles or distributes, or provides access to, any information pursuant to his employment or contract with the Commission. 
RWC 22-127

\section{POWER BURSTS IN NUCLEAR REACTORS}

\section{ABSTRACT}

It is shown that some of the properties of a power burst in a reactor are independent of either the feedback mechanism or the pile kinetics, and may be described quantitatively with no assumption other than that the pile kinetic equations are nonlinear. The theory refers primarily to the shape of the burst and is applicable chiefly to the faster transients observed in SPERT, KEWB and BORAX. The data which may be described theoretically in this manner include plots of maximum reactor power times period against the energy to peak of power burst (SPERT, KEWB), of total energy of burst against the period times the maximum power (BORAX), and of the burst width against period (SPERT)。In addition, use of the pile kinetic equations allows one to obtain a simple algebraic expression for the reactivity compensated at the time of peak power as a function of reciprocal period $\alpha$. This expression is in excellent agreement with experiment for the faster SPERT transients and exhibits the correct form of dependence on $\alpha$ for the slower transients. It is therefore pointed out that data which may be so simply described without reference to the feed-back mechanism do not furnish information about the nature of this mechanism. 
RWC 22-127

\section{INTRODUCTION}

When a positive excess reactivity $\mathrm{k}_{\mathrm{ex}}=\mathrm{k}_{0}$ is inserted into a reactor, the power will rise with $a$ period $\tau=\alpha^{-1}$ which is related to $k_{0}$ through the inhour formula:

$$
k_{0}=\alpha\left(\lambda+B \sum_{j} \frac{a_{j}}{\alpha+\lambda_{j}}\right)
$$

If inherent to the reactor is some effective shut-off mechanism, the reactor will begin to compensate for the initial reactivity as the power rises, until a maximum power is reached. If $\alpha$ and $k_{0}$ are sufficiently small, the reactor may have time to compensate for all of the initial reactivity by the time that peak power is reached, in which case it will continue to operate at this level. If on the other hand $\alpha$ is sufficiently large, only a fraction of the initial reactivity will be compensated at peak power but, despite this positive excess reactivity, the power may decrease sharply thereafter, with the reactor settling down later to some constant power much lower than that reached at the peak of the initial transient.

A number of power traces illustrating these phenomena are available from experiments with a wide variety of reactors. $1,2,3,4$ It is the purpose of this report to point out that some of the data so obtained may be derived theoretically by a very simple analysis that does not make any reference at all to the shut-off mechanism and, in some cases, does not even use details of the pile-kinetic equations. This means, conversely, that a study 
of these data does not yield any clue as to the nature of the phenomena responsible for shutoff.

2. EFFECTS INDEPENDENT OF FEED-BACK OR PILE-KINETICS.

Let us consider an initial transient which starts off with

a reciprocal period $\alpha$, so that initially $\emptyset=\emptyset_{1} e^{\alpha t}$, with $\mathbf{k}_{0}$, the initial excess reactivity, related to $\alpha$ by (1). Without making any postulate about the detalls of the shut-off mechanism, let us now suppose that this mechanism may be represented by some linear operator 0 , so that the feed-back equation is of the form $0 \mathrm{k}_{\mathrm{ex}}=\emptyset . \quad$ The operator 0 may involve differential or integral operators, or may be expressed as a Green's function

$$
k_{\text {ex }}=k_{0}-\underbrace{t}_{0} G\left(t-t^{i}\right) \emptyset\left(t^{i}\right) d t^{i}
$$

the negative sign being chosen to stress the fact that we are considering mechanisms which are in the direction to produce shutoff. As time develops, $\emptyset$ will begin to feed back into $k_{\text {ex }}$ a term proportional to $e^{\alpha t}$ and indeed when the feed-back equation has the form (2) we have, for $\emptyset=\emptyset_{1} e^{\alpha t}$,

$$
k_{e x}=k_{0}-\emptyset_{1} e^{\alpha t} i_{0}^{t} G(\eta) e^{-\alpha \eta} d \eta
$$

For sufficiently large $\alpha$, the appearance of $e^{-\alpha \eta}$ in the integrand causes the value of the integral to be relatively independent of the upper limit $t$, so that we may write

$$
k_{e x}=k_{0}-k_{1} e^{\alpha t} \quad\left(k_{1}=\text { const }<k_{0}\right)
$$


the condition under which this is a good approximation depending on the value of $\alpha$ and on the form of $G(\eta)$.

Due to the non-linear nature of the pile-kinetic equations, and in particular to the fact that $k_{e x}$ and $\emptyset$ appear multiplied together in these equations, the term $-k_{1} e^{\alpha t}$ in $k_{0}$, combined with the term $\emptyset=\emptyset_{1} e^{\alpha t}$, will begin to feed back into $\emptyset$ a term proportional to $e^{2 \alpha t}$ :

$$
\emptyset=\emptyset_{1} e^{\alpha t}-\emptyset_{2} e^{2 \alpha t} \quad\left(\emptyset_{2}<\emptyset_{1}\right)
$$

This represents the beginning of an expansion of $\varnothing$ in powers of $e^{\alpha t}$, and how far along the transient this expression will adequately represent the power trace will depend on the relative magnitude of the coefficients $\emptyset_{1}, \emptyset_{2}$, etc.

Without further assumptions, we now find that if we stop at the second term of this series, 1.e., use Eq. (4) to represent the power trace, a number of features of transients observed in SPERT, BORAX and KEWB may be described quantitatively. We use the following notation:

$$
\begin{aligned}
& \emptyset_{m}=\text { peak power } \\
& E\left(\emptyset_{m}\right)=\text { energy to peak power } \\
& t_{m}=\text { time of peak power } \\
& E_{\text {tot }}=\text { total energy of burst } \\
& B=\text { burst-width, } i_{\circ} e_{\circ} \text { width at half-maximum } \\
& z=e^{\alpha t_{m}} \\
& ;=\alpha^{-1}
\end{aligned}
$$


From (4) it follows by differentiation that

$$
z=\frac{\emptyset_{1}}{2 \varnothing_{2}}, \emptyset_{m}=\frac{\emptyset_{1}{ }^{2}}{4 \emptyset_{2}}
$$

Thus

$$
\emptyset_{\mathrm{m}}=\frac{1}{2} \emptyset_{1} \mathrm{z}=\frac{1}{2} \emptyset_{1} e^{\alpha t_{m}}
$$

or the maximum power is one-half of the initial power extrapolated to the time of maximum power. This result is in fair agreement with experiment in the faster SPERT and KEWB transients, as indicated in Table I. However, owing to the difficulty of estimating the extrapolated power at the time of peak power from a given trace, the relation (6) is not the most convenient one for testing the validity of the approximation (4). Characteristic data from such widely different reactors as BEPO and GODIVA are included for comparison.

TABLE I

\begin{tabular}{|l|c|c|c|c|c|c|}
\hline REACTOR & SPERT & SPERT & KEWB & KEWB & BEPO & GODIVA \\
\hline Period (ms) & 12 & 100 & 1.97 & 5.11 & 28,000 & 0.09 \\
\hline$\emptyset_{\mathrm{m}}(\mathrm{MW})$ & 520 & 13.3 & 550 & 128 & 6.8 & $\begin{array}{l}2.6 \times 10^{18} \\
\text { fissions } \mathrm{sec}^{-1}\end{array}$ \\
\hline$\frac{1}{2} \emptyset_{1} e^{\alpha \mathrm{t}_{\mathrm{m}}}$ & 460 & 13 & 580 & 118 & 15 & $\begin{array}{l}3 \times 10^{18} \\
\text { fissions sec }\end{array}$ \\
\hline
\end{tabular}


The energy to peak power is obtained by integrating Eq. (4)

$$
E\left(\emptyset_{m}\right)=\frac{1}{\alpha}\left[\emptyset_{1} z-\frac{1}{2} \emptyset_{2} z^{2}\right]=\frac{3}{8} \frac{\emptyset_{1}^{2}}{\alpha \emptyset_{2}}
$$

so that the relation between $E\left(\emptyset_{m}\right)$ and $\emptyset_{m}$ becomes independent of the parameters $\emptyset_{1}, \emptyset_{2}$,

$$
\sigma_{\mathrm{m}}=\frac{2}{3} \mathrm{E}\left(\emptyset_{\mathrm{m}}\right)
$$

This equation is compared with SPERT data in Fig. 1 (straight line labeled $\Xi=0$ ). Agreement with experiment is particularly good for the more energetic transients. Fig. 2 shows the same graph for KEWB. 5

A similar comparison with experiment may be made for BORAX where the available data are given in the form of a plot of $E_{\text {tot }}$ against $\tau \emptyset_{\mathrm{m}}$. Since expression (4) becomes zero for $t=t_{0}$ where $e^{\alpha t_{0}}=\frac{\emptyset_{1}}{\emptyset_{2}}$, the total energy of the burst is

$$
E_{t o t}=\frac{1}{\alpha}\left(\emptyset_{1} e^{\alpha t_{0}}-\frac{\emptyset_{2}}{2} e^{2 \alpha t_{0}}\right)=\frac{1}{2 \alpha} \frac{\emptyset_{1}^{2}}{\emptyset_{2}}
$$

Again the unknown parameters $\emptyset_{1}, \emptyset_{2}$ disappear if, using (5), we write this in the form

$$
E_{\text {tot }}=2 \tau \emptyset_{m}
$$

In Fig. 3, Eq. (9) is compared with experiment for BORAX data. 
The full curve is the empirical fit to the data previously published, ${ }^{6}$ and the dashed curve is Eq. (9). The data on this plot are seen to be independent of the water temperature and indeed from the above arguments they are seen to be independent of the shut-off mechanism in a very general way. The agreement does not necessarily imply, however, that Eq. (4) is an adequate representation of the power throughout the whole transient. Departure of Eq. (4) from the observed power at points where the power has dropped to less than $1 \%$ of its peak value would generally produce a negligible error in the total energy of the excursion.

From Eq. (4) it also follows that if $t_{1}, t_{2}$ are the times at which the power is equal to $\frac{1}{n}$ times the maximum power, then if $y=e^{\alpha t_{1}}$ or $e^{\alpha t_{2}}$,

$$
\emptyset_{2} \mathrm{y}^{2}-\emptyset_{1} \mathrm{y}+\frac{\emptyset_{\mathrm{m}}}{\mathrm{n}}=0
$$

Thus from (5),

$$
\left.y=\frac{\emptyset_{1}}{2 \emptyset_{2}}: 1 \pm \sqrt{1-\frac{1}{n}}\right)
$$

so that

$$
\left(t_{2}-t_{1}\right)=\tau \ln \frac{1+\sqrt{1-\frac{1}{n}}}{1-\sqrt{1-\frac{1}{n}}}
$$

the parameters $\emptyset_{1}, \emptyset_{2}$ again canceling out. For $n=2$, this is the burst width 


$$
B=\tau \ln \left(\frac{\sqrt{2}+1}{\sqrt{2}-1}\right)=1.76 \tau
$$

Fig. 4 shows SPERT normalized power traces plotted against the time measured in periods. For the faster transients ( $\check{Z} \leq 35 \mathrm{~ms})$ the agreement with Eq. (11) is within the accuracy of observation. For $n=10$, Eq. (10) gives $t_{2}-t_{1}=3.8$ periods as compared to the experimentally observed 3.9 periods for the faster transients, and for $n=100$ one obtains 6 periods from Eq. (10), as opposed to 7 periods observed.

It is interesting to examine the effect of an extra term added to (4):

$$
\emptyset=\emptyset_{1} e^{\alpha t}-\emptyset_{2} e^{2 \alpha t}+\div \frac{\emptyset_{2}^{2}}{\emptyset_{1}} e^{3 \alpha t}
$$

where $\dot{E}$ is a dimensionless parameter, and the coefficient of the last term has been written in the form $\leqslant \frac{\emptyset_{2}^{2}}{\emptyset_{1}}$ for conven-

ience. It then follows that for $\epsilon>\frac{1}{3}$ the last term is so large that the power does not reach a maximum, but continues to increase monotonically. As long as we consider only the three terms of (8) we are therefore restricted to the range $0<\leq<\frac{1}{3}$ for any mechanism that produces shut-off. The effect of the extra term with its maximum value $\epsilon=\frac{1}{3}$ is shown in Figs. 1 and 2 , the slope being $\frac{6}{11}$ instead of $\frac{2}{3}$. For any experimental point lying below the curve $\epsilon_{:}=\frac{1}{3}$ in this figure the correspond- 
ing transient cannot be described in accurate detail all the way up to peak power by an expression of the form (12). For $0 \leq \epsilon \leq \frac{1}{3}$, Eq. (7) is then replaced by

$$
\tau \emptyset_{\mathrm{m}}=E\left(\emptyset_{\mathrm{m}}\right)\left[\frac{6 \epsilon-1+\sqrt{1-3 \epsilon}}{8 \epsilon-\frac{5}{6}(1-\sqrt{1-3 \epsilon})}\right]
$$

For $\epsilon \leq \frac{1}{4}$, expression (12) drops to zero after a time

lag beyond peak power given by

$$
\tau \ln \left[\begin{array}{ll}
\frac{3}{2} & \frac{1-\sqrt{1-4 \epsilon}}{1-\sqrt{1-3 \epsilon}}
\end{array}\right]
$$

i.e., $1.1 \tau$ for $\epsilon=\frac{1}{4}$, as compared with .69 $\tau$ for $\epsilon=0$. Reference to Figure 5 then shows that for $\epsilon<\frac{1}{4}$ the downward side of the transient as given by (12) is almost indistinguishable from the dotted curve for $\epsilon=0$. As $\epsilon$ increases from $\frac{1}{4}$ to $\frac{1}{3}$, however, the last term rapidly dominates the expression (12) beyond peak power. Thus for $\epsilon=.3$ the curve reaches a minimum of 0.8 only .65 periods after peak power, and thereafter rises very rapidly. The shape of the transients for longer periods is illustrated in Figure 6 for KEWB, SPERT and BEPO. The approximate shape of a characteristic GODIVA trace, is given on the same diagram. Comparison with Figure 5 shows that no cholce of $\epsilon$ will allow Equation (12) to represent such transients beyond peak power.

We should therefore expect that Eqs. (10), (11) are of less general validity than Eqs. (6), (7) which refer only to times 
up to peak power. This is illustrated in Figure 7 in which Eq. (11) is compared with experiment for GODIVA. The case $\epsilon=0$ gives the right order of magnitude for the burst width as given by the dotted line, although naturally this simple analysis cannot compete with the detailed calculations of Stratton and Colvin (full curve).

3. EFFECTS DEPENDING ON PILE-KINETICS

All of the conclusions drawn up to this point are independent both of the shut-off mechanism and the details of the pilekinetic equations, other than the fact that the latter are nonlinear. If now we use Eq. (4) in the pile-kinetic equations we may compute the excess reactivity as a function of time during the course of a transient in terms of the two parameters $\emptyset_{1}, \emptyset_{2}$. The analysis is simplified by using the form of the pile-kinetic equations in which the precursor densities have been eliminated algebraically. 7,8 The expression so obtained provides an accurate description of the time-dependence of the reactivity for times during which (4) is valid. If this function is evaluated at the time of peak power and subtracted from the initial reactivity, one obtains the following expression for the reactivity compensated by the reactor at the time of peak power:

$$
k_{c}\left(t_{m}\right)=\alpha\left[\ell+B \sum_{j} \frac{a_{j} \lambda_{j}}{\left(\alpha+\lambda_{j}\right)\left(2 \alpha+\lambda_{j}\right)}\right]
$$


Once again the unknown parameters $\emptyset_{1}, \emptyset_{2}$ and the time $t_{m}$ of peak power cancel out. We note that as $\alpha \rightarrow \infty, \mathrm{k}_{\mathrm{c}} \rightarrow \mathrm{k}_{\mathrm{o}}-\beta$, each tending to $\alpha \ell$ in this 1imit. As $\alpha \rightarrow 0$, on the other hand, $k_{c} \rightarrow k_{o}$ (each tending to $\alpha\left(l+B \sum \frac{a_{j}}{\lambda_{j}}\right)$ i.e., all of the initial reactivity is compensated by the reactor at the time of peak power as would be expected for an infinitesimally slow transient.

The function (13) is graphed in Figure 8 and the general form of the dependence of $k_{c}\left(t_{m}\right)$ on $\alpha$ is seen to be given even in the approximation $\epsilon=0$. For $\epsilon=\frac{1}{3}$, one obtains a correction term

$$
k_{c}\left(t_{\left.m_{\epsilon}\right)}=k_{c}\left(t_{m}\right)=\alpha_{\epsilon=0}^{2}+\sum_{j} \frac{a_{j} \lambda_{j}}{\left(\alpha+\lambda_{j}\right)\left(2 \alpha+\lambda_{j}\right)\left(3 \alpha+\lambda_{j}\right)}\right.
$$

which vanishes for large and small values of $\alpha$ and which improves the agreement with experiment for the slower transients.

We therefore see that the general form, and indeed many of the details of the observed data on reactivity compensated at the time of peak power as a function of $\alpha$, may be described without any reference to the mechanism of shut-off and in particular that the minimum around $8 \mathrm{sec}^{-1}$ and the maximum near $0.1 \mathrm{sec}^{-1}$ are properties of the curve that arise solely from delayed neutron effects. If a reactor is going to rise in power and come to a maximum, one can therefore be sure that at that maximum the 
reactor has already compensated for some of the reactivity that was inserted originally and that this amount of compensated reactivity may be computed at least approximately for any reactor rising with any period.

In addition to the error introduced by terminating the serles for $\emptyset$ according to Eq. (4) ( $E=0$ ) or Eq. (8) ( $\left.\epsilon \leq \frac{1}{3}\right)$, a related error is introduced by the approximation, noted in (3), (4), that $k_{1}<<k_{0}, \emptyset_{2}<<\emptyset_{1}$. This error becomes increasingly important for slower transients. To estimate 1 ts effect, we note that, according to (3), (4), $k_{e x}$ does not approach $k_{0}$, and $\emptyset$ does not approach $\emptyset_{1} e^{\alpha t}$ except in the limit $t \rightarrow-\infty$. We shall choose a finite time, labeled $t=0$, at which $k_{e x}=k_{0}$ given by (1) (c.f. Eq. (2)) and at which $\emptyset=\emptyset_{1} \mathrm{e}^{\alpha \mathrm{t}}$, replacing (3) and (4) by

$$
\begin{array}{ll}
k_{\text {ex }}=k_{0}+k_{1}-k_{1} e^{\alpha t} & (t>0) \\
k_{\text {ex }}=k_{0} & (t<0) \\
\emptyset=\emptyset_{1} e^{\alpha t}+\emptyset_{2}-\emptyset_{2} e^{2 \alpha t} & (t>0) \\
\emptyset=\emptyset_{1} e^{\alpha t} & (t<0)
\end{array}
$$

The choice $t=0$ is arbitrary and introduces into the description of the transient an extra parameter ( $e_{.} g_{\circ}$, the time $t_{m}$ from zero to peak power) which, 11ke $\leqq$ in Eq. (12), does not cancel out when the equations corresponding to (7), (9), (10) and (13) are derived. Writing $y=e^{\alpha t_{m}}$, we find that Eq. (7) is now replaced by 
RWC 22-127

$$
\frac{2}{3} E\left(\emptyset_{m}\right)=\tau \emptyset_{m}\left[1+\frac{2}{3\left(1+y^{2}\right)}(\ell n y-1)\right]
$$

the correction term being a maximum, for given $\emptyset_{m}$, when

$$
l_{\mathrm{ny}}=\frac{3}{2}+\frac{1}{2 \mathrm{y}^{2}}
$$

or $y \div 4.5$. Although for $\alpha t_{m}>1$ (i.e., $\ell_{n y}>1$ ) this is in the correct direction to describe the points on Figure 1 lying below the curve $\epsilon=0$, the maximum value of the correction is less than $2 \%$, and hence may be neglected. 


\section{REFERENCES}

1. F. Schroeder, S. G. Forbes, W. E. Nyer, F. L. Bentzen and G. O. Bright, Nuc. Sci. and Eng. 2. 96-115 (1957).

2. C. Rogers McCullough, "Safety Aspects of Nuclear Reactors," Van Nostrand (1957).

3. W. R. Stratton and T. H. Colvin, "Analysis of Prompt Excursions in Simple Systems, "LASL N-2-597 (1957).

4. D. V. Wordsworth, "An Analysis of Power, Temperature and Reactivity Transients in BEPO." AERE-E/R-1245 (1953).

5. The author wishes to thank J. W. Flora for providing him with power traces of KEWB transients.

6. Ref. 2 p. 219. Fig. 9.15.

7. W. A. Horning and H. C. Corben, "Theory of Power Transients in the SPERT I Reactor," Part I, (Jan. 1958). IDO-16434.

8. H. C. Corben, D. B. Langmuir and R. S. Margulies, "Theory of Small Oscillations in Boiling Water Nuclear Reactors," RWC-22-122 (June 1958). 


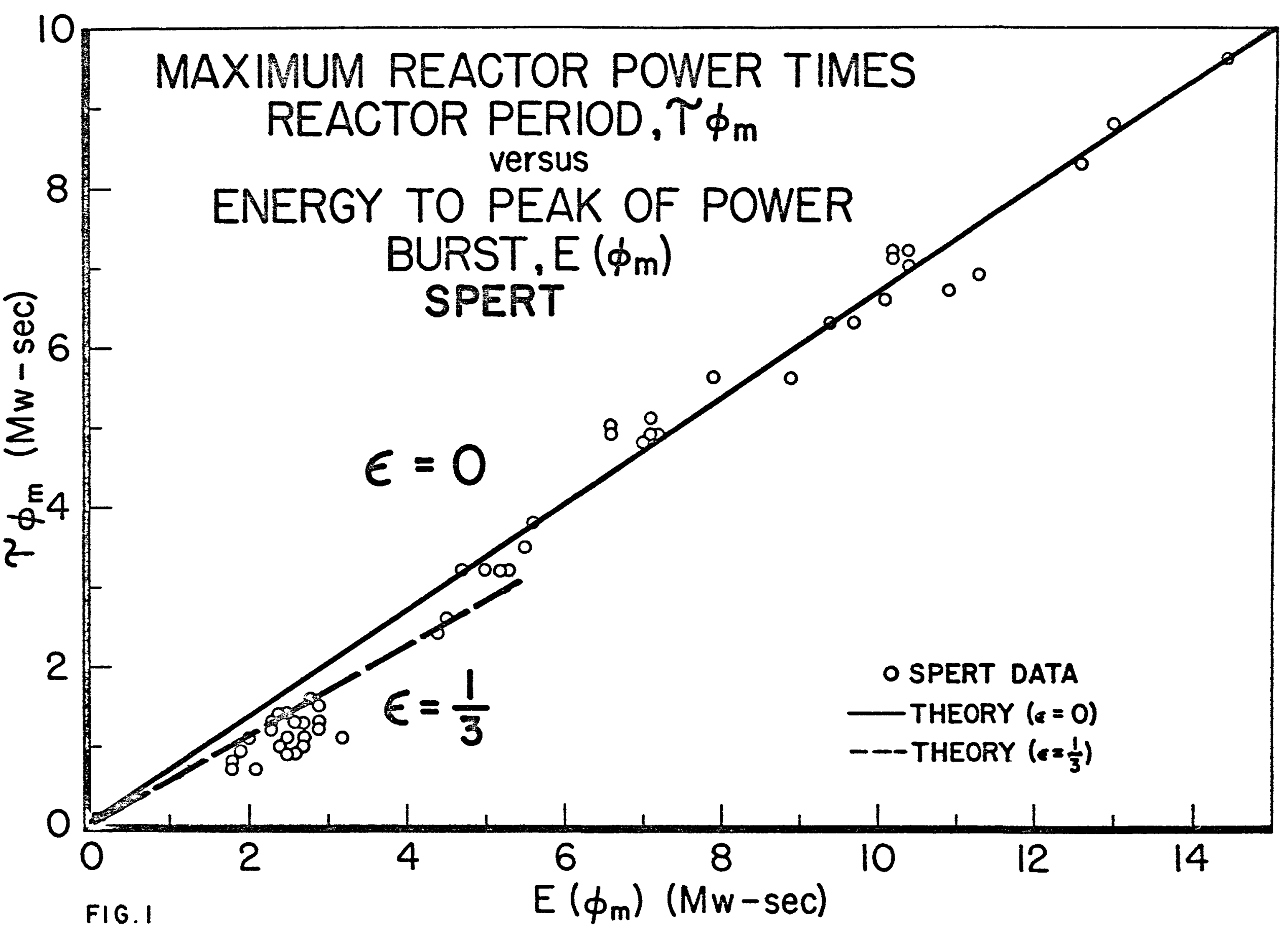




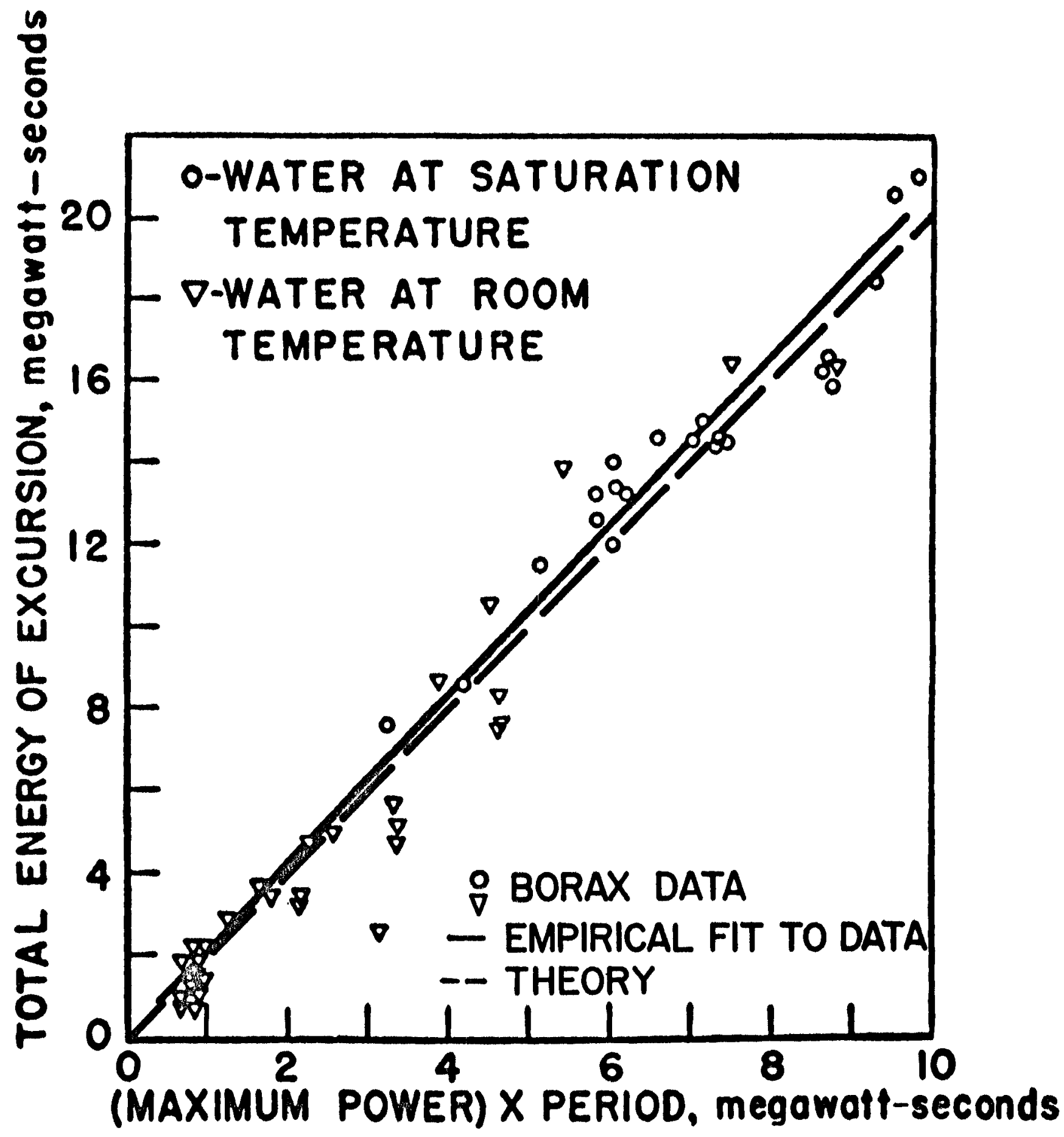

FIG. 3 


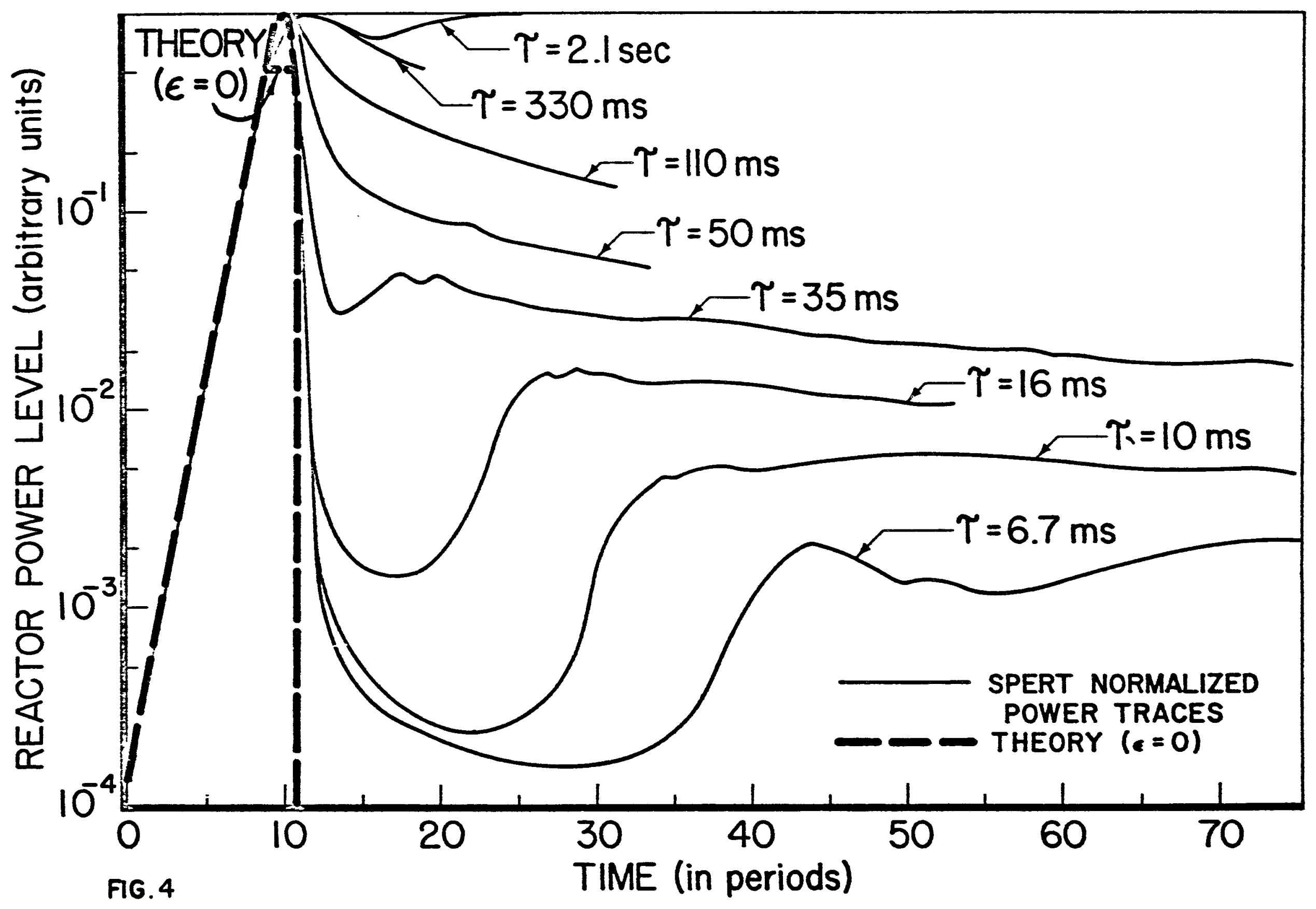




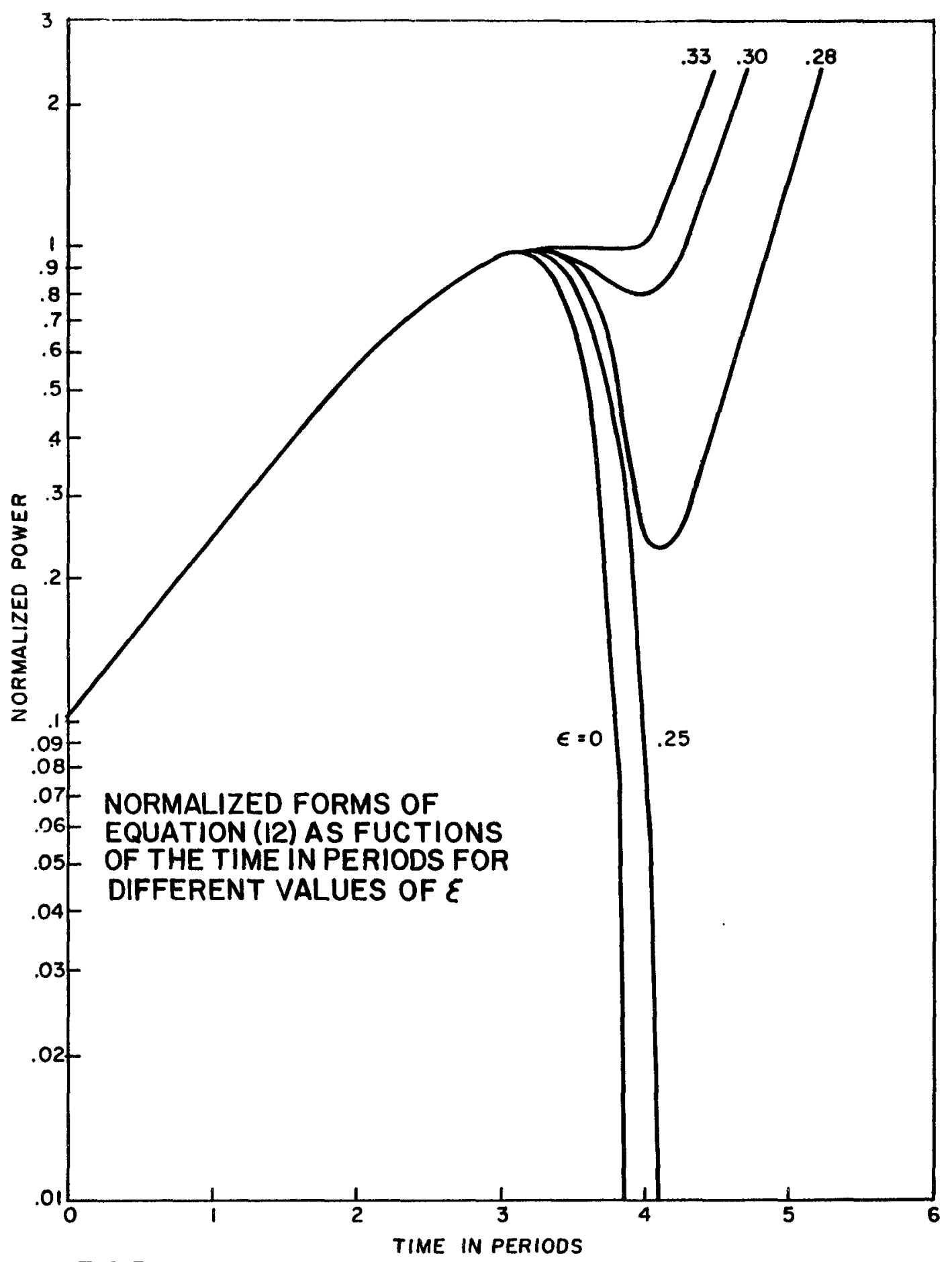

FIG.5 


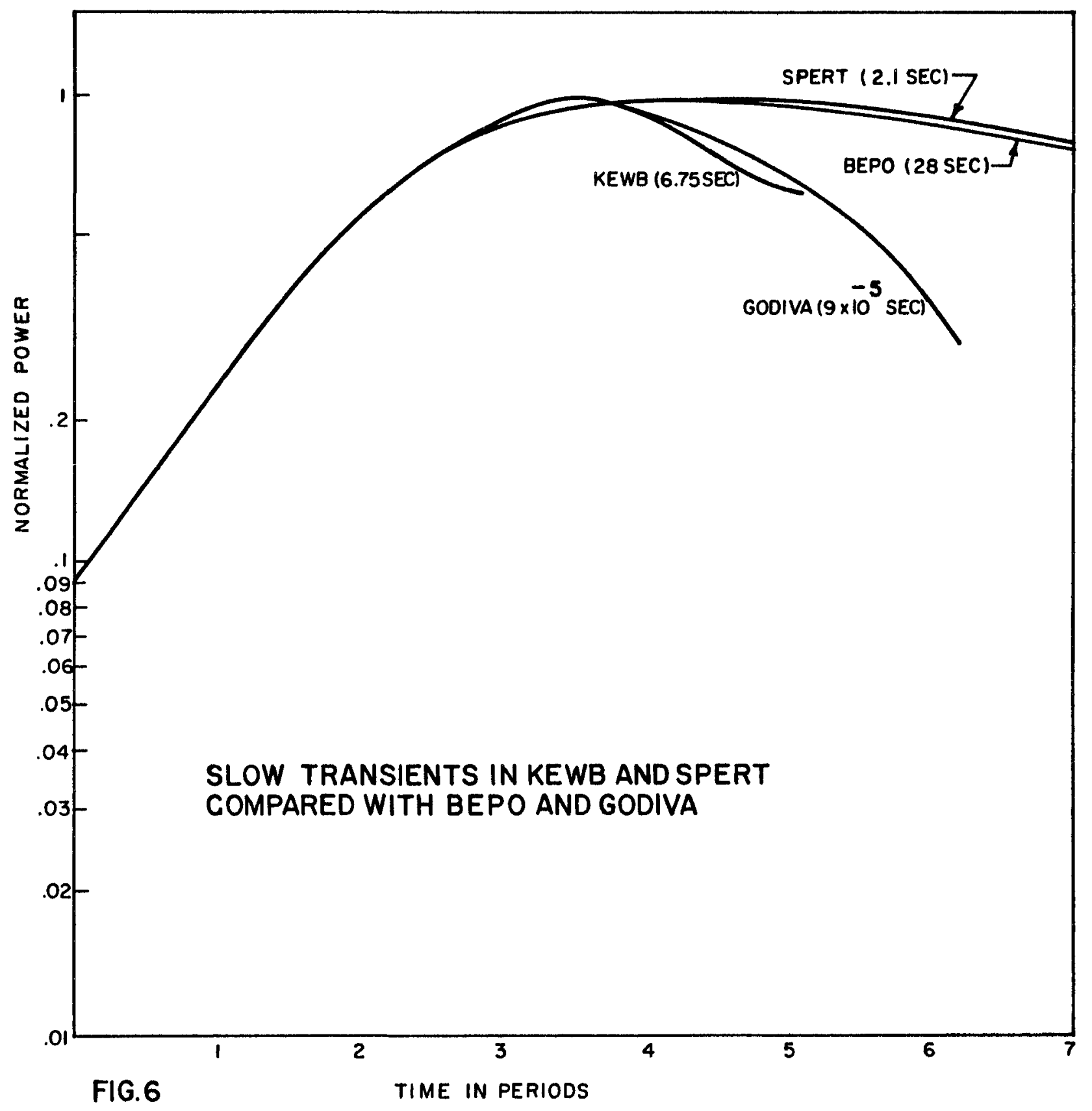




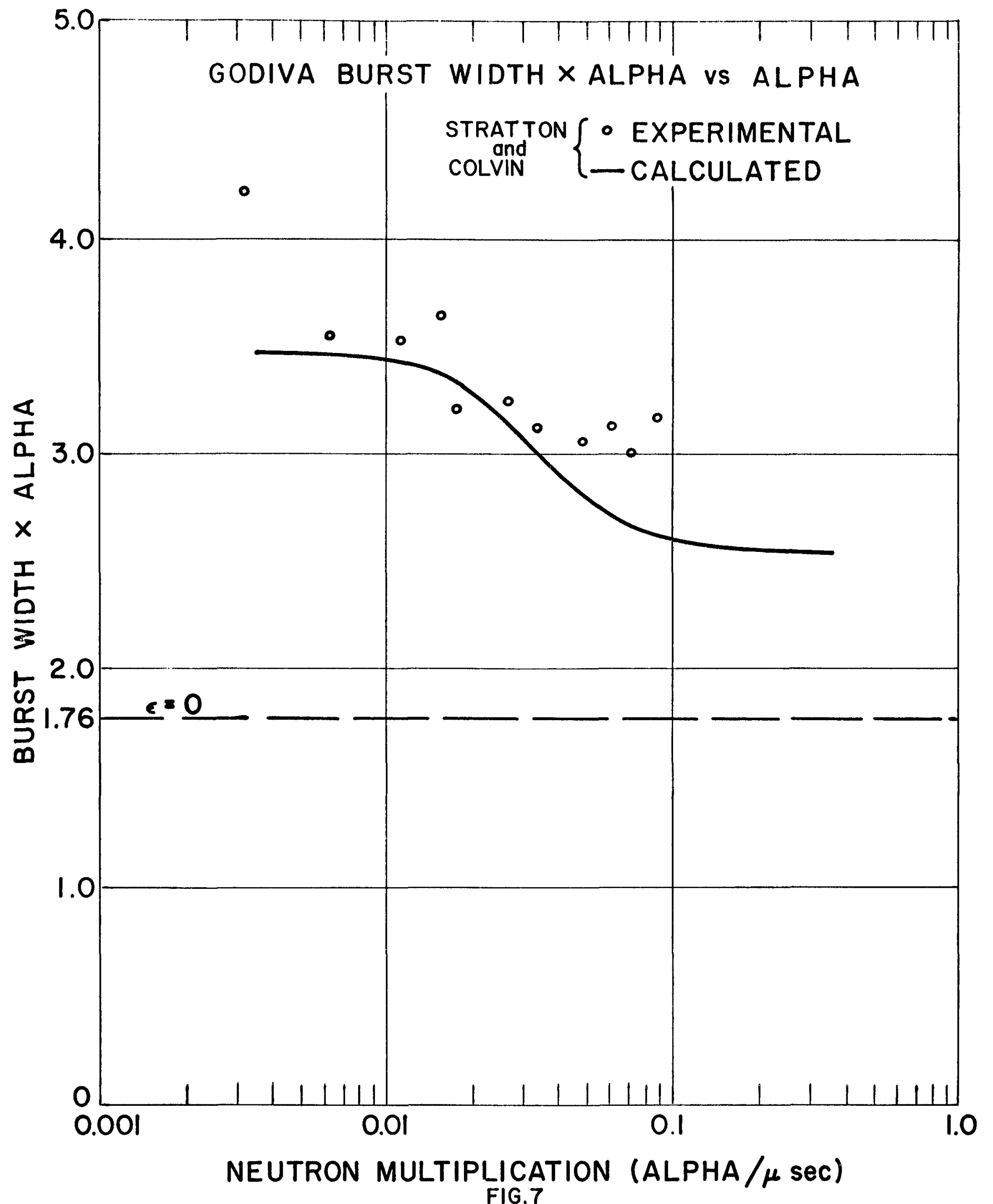




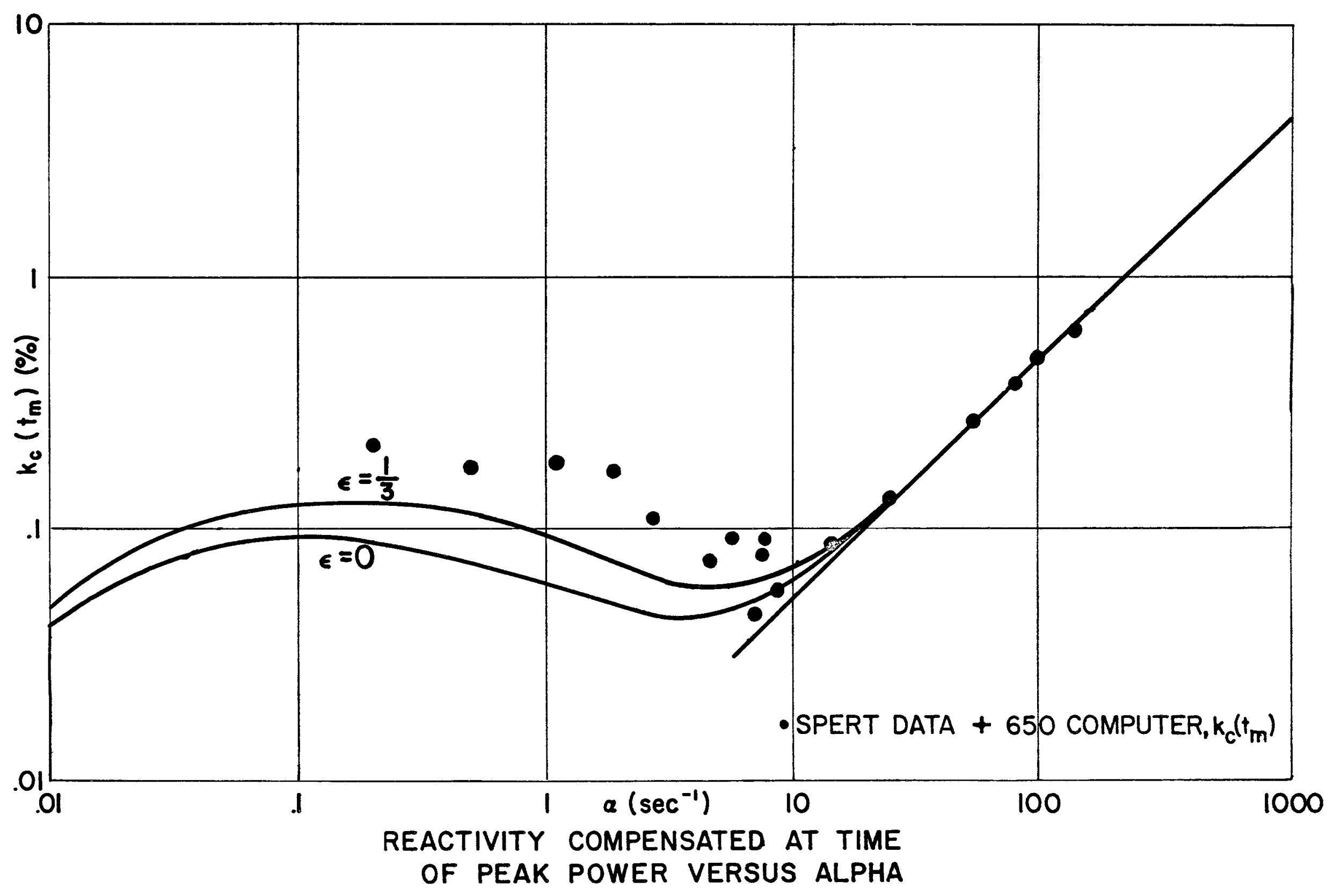

FIG. 8 